\title{
Condições clínicas de saúde em idosos submetidos à amputação de membros superiores e inferiores
}

\author{
Clinical health conditions in the elderly submitted to upper and lower limb amputation \\ Afecciones clínicas de salud en ancianos sometidos a amputación de miembros superiores e \\ inferiores
}

Kimberly Mayara Gouveia Bezerra ORCID: https://orcid.org/0000-0001-8186-1731

Universidade Federal de Pernambuco, Brasil

E-mail:kim.mgouveia@gmail.com

Márcia Carréra Campos Leal

ORCID: https://orcid.org/0000-0002-3032-7253

Universidade Federal de Pernambuco, Brasil

E-mail:marciacarrera@hotmail.com

Ylkiany Pereira de Souza

ORCID: https://orcid.org/0000-0001-9710-9202

Hospital Getúlio Vargas, Brasil

E-mail: ylkiany@hotmail.com

Ana Paula de Oliveira Marques

ORCID: https://orcid.org/0000-0003-0731-8065

Universidade Federal de Pernambuco, Brasil

E-mail: ana.marques@ufpe.br

Ana Carolina de Carvalho Leandro

ORCID: https://orcid.org/0000-0001-5884-5901

Universidade Federal de Pernambuco, Brasil

E-mail:ana96.leandro@gmail.com

Maria Gabryelle Jatobá Pereira de Brito

ORCID: https://orcid.org/0000-0001-7395-6933

Universidade Federal de Pernambuco, Brasil E-mail: gabryelle.brito@ufpe.br

Cayo Cesar da Silva

ORCID: https://orcid.org/0000-0002-1849-7188 Universidade Federal de Pernambuco, Brasil E-mail: cayoscesar@gmail.com

Denise Maria Nascimento de Lima ORCID: https://orcid.org/0000-0003-2361-4892

Universidade Federal de Pernambuco, Brasil

E-mail:denise.lima@ufpe.br

Crislayne Maria Berto

ORCID: https://orcid.org/0000-0003-3022-2117

Universidade Federal de Pernambuco, Brasil E-mail:crislayneberto@gmail.com

Samara Souza Vieira

ORCID: https://orcid.org/0000-0002-7531-0711

Universidade Federal de Pernambuco, Brasil E-mail: samara.souza@ufpe.br

Fernanda Oliveira Santos

ORCID: https://orcid.org/0000-0001-8407-8830 Universidade Federal de Pernambuco, Brasil E-mail: fernanda.osantos@ufpe.br

Leiliane Moraes dos Santos Silva

ORCID: https://orcid.org/0000-0003-4882-1628 Universidade Federal de Pernambuco, Brasil E-mail: leiliane.moraes@ufpe.br

Fernando Arthur Alves da Silva ORCID: https://orcid.org/0000-0001-8883-6037 Universidade Federal de Pernambuco, Brasil E-mail: fernando.arthur@ufpe.br

Everton Ferreira Rodrigues Nascimento

ORCID: https://orcid.org/0000-0002-4731-0466

Universidade Federal de Pernambuco, Brasil

E-mail:everton.rodriguesnascimento@ufpe.br 


\begin{abstract}
Resumo
Introdução: A amputação consiste na remoção de um membro ou um segmento dele, podendo ser maior ou menor dependendo do local acometido, e a presença de comorbidades como diabetes e hipertensão arterial são consideradas fatores de risco para sua realização. Objetivo: Conhecer quais as características clínicas de saúde mais prevalentes em idosos submetidos a amputações em um serviço de referência, com o intuito de ajudar na elaboração de ações de saúde para melhor atender essa população. Metodologia: Trata-se de um estudo descritivo, quantitativo, retrospectivo de corte transversal, realizado com 418 idosos amputados em um hospital de grande porte localizado na cidade de Recife (PE). Resultado: As comorbidades mais prevalentes na amostra estudada foram Hipertensão Arterial Sistêmica (HAS) (83,7\%), Diabetes Mellitus (DM) (83,3\%), ex-tabagismo (37,0\%), tabagismo (28,5\%) e doença renal $(28,1 \%)$. A Isquemia Crítica de Membro Inferior Direito (ICMID) (31,5\%), a Isquemia Crítica de Membro Inferior Esquerdo (ICMIE) $(28,3 \%)$ e gangrena úmida $(14,4 \%)$ foram as principais causas de amputação. Conclusão: Espera-se que o estudo possa corroborar para a elaboração de estratégias educativas e preventivas, bem como contribuir para que ações possam ser direcionadas para esse público a fim de manter uma assistência adequada com foco no controle e tratamento dos fatores causais das amputações, objetivando melhoria da qualidade de vida.
\end{abstract}

Palavras-chave: Amputação; Idoso; Extremidade superior; Extremidade inferior.

\begin{abstract}
Introduction: Amputation is the removal of a limb or a segment of it, which can be larger or smaller depending on the affected site, and the presence of comorbidities such as diabetes and hypertension are considered risk factors for its realization. Objective: To know what are the most prevalent clinical health characteristics in elderly patients undergoing amputations in a reference service, in order to help in the development of health actions to better serve this population. Methodology: This is a descriptive, quantitative, retrospective cross-sectional study, carried out with 418 elderly amputees in a large hospital located in the city of Recife (PE). Results: The most prevalent comorbidities in the studied sample were Systemic Arterial Hypertension (SAH) (83.7\%), Diabetes Mellitus (DM) (83.3\%), ex-smoking (37.0\%), smoking $(28.5 \%)$ and kidney disease $(28.1 \%)$. Right Lower Limb Critical Ischemia (ICMID) (31.5\%), Left Lower Limb Critical Ischemia (ICMIE) (28.3\%) and wet gangrene (14.4\%) were the main causes of amputation. Conclusion: It is hoped that the study can support the development of educational and preventive strategies, as well as contribute to actions that can be directed to this audience in order to maintain adequate care focused on the control and treatment of the causal factors of amputations, aiming to improve the quality of life.
\end{abstract} Keywords: Amputation; Aged; Upper extremity; Lower extremity.

\title{
Resumen
}

Introdución: La amputación consiste en la remoción de un mienbro o un segmento de él, puede ser más grande o más pequeno dependiendo de lugar afectado, y la presencia de comorbilidades como la diabetes y la hipertensión arterial se consideran factores de riesgo para su realización. Objetivo: Conoce cuáles son las características clínicas de salud más prevalentes en los ancianos sometidos a amputaciones en un servicio de referencia, con la intención de ayuda con la elaboración de acciones de salud para servir mejor esta población. Metodologia: Se trata de un estudio descriptivo, cuantitativo, retrospectivo de corte transversal, realizado con 418 ancianos amputados en un gran hospital de la ciudad de Recife (PE). Resultado: Las comorbilidades más prevalentes en la muestra estudiada fueron Hipertensión Arterial Sistémica (HAS) (83,7\%), Diabetes Mellitus (DM) (83,3\%), ex-fumador (37,0\%), fumador (28,5\%), y enfermedad renal $(28,1 \%)$. La Isquemia Crítica del Miembro Inferior Derecho (ICMID) (31,5\%), Isquemia Crítica del Miembro Inferior Izquierdo (ICMII) (28,3\%), y la gangrena húmeda (14,4\%) fueron las principales causas de amputación. Conclusión: Se espera que el estudio pueda corroborar para la elaboración de estratégias educativas y preventivas, así como contribuir a qué acciones se dirijan a este público para mantener una atención adecuada centrada en el control y tratamiento de los factores causales de las amputaciones, con el objetivo de mejorar la calidad de vida.

Palabras clave: Amputación; Anciano; Extremidad superior; Extremidad inferior.

\section{Introdução}

A transição demográfica trouxe consigo alterações no perfil epidemiológico, pois as pessoas idosas começaram a apresentar doenças crônicas não transmissíveis (DCNT) e incapacidades decorrentes dela, como a amputação, com números crescentes ao longo dos anos. Nesse contexto, em 2018, foram registradas mais de 59 mil amputações no país, das quais 2.694 ocorreram no estado de Pernambuco, colocando o estado em oitavo lugar em número de amputações (Datasus, 2019).

A amputação consiste na remoção de um membro ou um segmento dele, a qual pode ser dividida em amputações maiores e menores, sendo as primeiras aquelas que acometem boa parte do membro, como transtibiais e transfemurais, enquanto que as segundas ocorrem em um nível mais distal, como dedo, punho, tarso ou mão (Brasil, 2013; Jesus-Silva, et al., 2017). 
Evidencia-se na literatura diferenças significativas entre amputações decorrentes de condições clinicas e traumáticas. A primeira ganha destaque por ser a mais prevalente, atingir principalmente pessoas de idades mais avançadas e afetarem os membros inferiores, já as amputações traumáticas afetam mais jovens do sexo masculino e têm os acidentes de trabalho e automobilísticos como os maiores causadores (Montiel \& Vargas \& Leal, 2012).

Os fatores de risco para amputação estão muito relacionados com o nível de amputações, menores ou maiores, evidencia-se em muitos estudos que idade avançada, Acidente Vascular Encefálico prévio (AVE), amputação prévia, sepse, isquemia e anemia estiveram mais relacionados às amputações maiores, enquanto diabetes, presença de pulso distal e neuropatia instalada estiveram mais relacionados às amputações menores, bem como observou que o principal fator que determina para a evolução entre amputação maior e menor era a condição inicial de revascularização do membro. A presença de comorbidades como diabetes, hipertensão arterial, doença renal, doença vascular periférica, dislipidemia, obesidade, sedentarismo são considerados fatores de risco para a amputação (Jesus-Silva, et al., 2017; Almeida, et al., 2013).

O tempo de comprometimento do membro, as características do indivíduo, aumento do número de comorbidades podem aumentar o risco de amputações maiores e muitas vezes levar ao óbito (Rolim, et al., 2015).

Além disso, as amputações de etiologia não traumática prevalecem no Brasil, principalmente no sexo masculino, isso pode ser justificado pelo fato das mulheres procurarem mais assistência à saúde e terem uma preocupação maior com sua saúde. Entretanto, estudos apontam que os homens são mais submetidos a procedimentos de amputação, mas a maior taxa de mortalidade está entre as mulheres amputadas (Seidel, et al., 2009; Peek, 2011; Dillon, et al., 2017).

É importante salientar que a amputação é um problema de saúde pública, que afeta mais e mais pessoas a cada ano, e grande parte das causas das amputações poderiam ser evitadas com medidas de promoção e prevenção a saúde. No entanto, cada vez mais pessoas vêm sendo amputadas, com dificuldade na socialização, trabalho laboral, perda da independência e, por conseguinte, comprometimento na qualidade de vida (Senefonte, et al., 2012).

Dentre as doenças crônicas que mais estão relacionadas à amputação, está o Diabetes Mellitus (DM). Segundo a Sociedade Brasileira de Angiologia e de Cirurgia Vascular (SBACV, 2018), 80\% das amputações não traumáticas ocorrem em pacientes diabéticos. Ao investigar estudos que avaliaram as condições clínicas de pacientes amputados, em grande quantitativo deles os indivíduos apresentavam um ou mais destes problemas: diabetes, hipertensão arterial, doença renal ou alto risco de doença renal, vasculopatias, cardiopatia, obstrução arterial crônica ou aguda, dislipidemia e tabagismo, sendo que muitos deles são determinantes do óbito após a cirurgia de amputação (Chanliam, et al., 2032; Oliveira, et al., 2017; Leandro, et al., 2018).

Já se sabe que a principal causa das amputações não traumáticas no membro inferior advém de patologias vasculares, como a doença vascular periférica combinada ou não com o DM, além de processos isquêmicos envolvidos em vasculopatias, e ocorrem frequentemente em indivíduos idosos. Devido ao número elevado de comorbidades entre esse grupo populacional, o risco para amputações torna-se aumentado (Santos, et al., 2018). Desse modo, o controle dessas doenças, bem como um olhar voltado as especificidades do processo de envelhecimento torna-se fundamental para a prevenção desse agravo.

Além disso, o último estudo sobre Carga Global de Doença (GBD - Global Burden of Diseases) alerta para o aumento de anos de vida sadia perdidos. O estudo de Santos, et al (2018), evidencia a preocupação com o impacto dos anos de vida perdidos ao longo das próximas duas décadas, dando maior enfoque para DM e suas complicações, como por exemplo, neuropatia diabética, retinopatia diabética, doença renal crônica (DRC) e amputações. As projeções feitas pela GBD mostram que os indivíduos com DCNT viverão mais, porém com sequelas de AVC, diálise e amputação por DM.

Não obstante o envelhecimento ser visto como uma das grandes conquistas alcançadas, não se tem ainda uma preparação adequada para um país de longevos, que garanta a eles qualidade de vida (WHO, 2005). O sistema de saúde brasileiro ainda não está organizado para prestar uma assistência em tempo hábil para todos os usuários, assim, a longa espera por um atendimento médico acaba prejudicando a condição de saúde do paciente, a exemplo disso, são pacientes com fatores de risco para amputação 
que quando não têm acesso aos serviços de saúde com facilidade, acabam sendo admitidos nos serviços de maior complexidade sem condições para alternativas de salvar o membro (como a revascularização) levando a uma amputação (Schmidt, et al., 2011).

Percebe-se então que muito ainda tem que ser feito para que a presença de doenças crônicas não seja um fator que predomine e afete tanto a qualidade de vida das pessoas, que elas possam ter uma assistência à saúde integral com cuidados voltados para a promoção da saúde. E que quando presente alguma doença crônica, esta possa ser controlada adequadamente por uma equipe de saúde qualificada em um sistema de saúde preparado para idosos.

Diante disso, é fundamental conhecer melhor o perfil de saúde de cada localidade e ajudar na elaboração de ações de saúde para uma adequada atenção aos fatores de riscos para o desenvolvimento de amputações, visando preveni-las ou evitá-las, e, através dos dados estudados, as ações possam ser direcionadas da melhor forma possível a manter uma assistência adequada para os idosos com foco no controle e tratamento dos fatores causais das amputações.

Assim, o objetivo do estudo foi conhecer quais as características clínicas de saúde mais prevalentes em idosos submetidos a amputações em um serviço de referência, com o intuito de ajudar na elaboração de ações de saúde para melhor atender essa população.

\section{Metodologia}

Tratou-se de um estudo descritivo, quantitativo, retrospectivo de corte transversal, realizado no Hospital Getúlio Vargas, localizado no município do Recife-PE.

Visto isso, entende-se como estudo descritivo aquele que tem por objetivo a determinação da distribuição de doenças ou condições clínicas que estão relacionadas à saúde, de forma a contextualizá-las ao tempo, lugar e características individuais de cada pessoa. Associado a isso, por meio dos estudos quantitativos, interpretamos os resultados através da análise de dados, indicadores e tendências observáveis. De forma complementar, compreende-se como retrospectiva a pesquisa que relaciona a história pregressa do indivíduo a sua patologia atual, que busca identificar os fatores relacionados à condição clínica do paciente e analisar fatores de risco para uma determinada população (Lima-Costa \& Barreto, 2003).

A população selecionada para o estudo foi constituída por prontuários de pacientes idosos (acima de 60 anos) que foram submetidos à amputação entre os anos de 2016 e 2018.

Utilizando os livros de documentação de cirurgias do centro cirúrgico do hospital, foram obtidas todas as amputações realizadas entre os anos de 2016 a 2018, que totalizaram 1310, sendo 96 excluídas logo de início por já está especificado no livro idade menor de 60 anos e uma pela ausência do registro de identificação do paciente impossibilitando encontrá-lo no sistema do hospital.

Logo após, com os registros de identificação dos pacientes no SAME foi obtido o acesso ao sistema eletrônico de pacientes do hospital, onde excluiu- se 412 pacientes que se submeteram a amputações, mas tinham a idade inferior a 60 anos e 90 pacientes que não foram encontrados no sistema, nem com a utilização do nome completo, nem com o registro de identificação, restando no final 666 idosos para realizar a busca no arquivo por prontuário. Destes, 235 não foram encontrados e 13 não constavam amputação no prontuário, apenas a realização de outros procedimentos como desbridamento e regularização de coto.

Vale salientar que não foi possível estabelecer a idade de 90 pessoas amputadas, pela falta do registro da idade no livro de cirurgias e por não encontrar o indivíduo no sistema eletrônico, o que pode ter sido decorrido de erro ao anotar o registro e o nome do paciente. Assim a amostra final totalizou 418 idosos amputados.

Idosos que estavam cadastrados nos Serviços de Referência no período estabelecido para o estudo (2016 a 2018); de ambos os sexos; com idade mínima de 60 anos (condição que define a pessoa idosa, de acordo com a Lei n $8842 / 94$, que trata 
sobre a Política Nacional do Idoso). Foram excluídos os prontuários de idosos com ilegibilidade de informações e/ou incompletos.

A coleta de dados foi iniciada no mês de novembro de 2019 e finalizada em fevereiro de 2020 e foi realizada em dois momentos, a primeira através dos registros de amputações de cirurgias no bloco cirúrgico e a segunda no Serviço de Arquivo Médico e Estatística (SAME) do Hospital, através do prontuário.

Utilizou-se um formulário próprio, composto por dados das condições clínicas de saúde dos idosos amputados (morbidades referidas em prontuário) e etiologia da amputação baseado nos objetivos e itens disponibilizados no serviço e aplicado por três pesquisadores previamente treinados. A coleta foi realizada a partir de dados do centro cirúrgico do hospital e do serviço de arquivo médico e estatístico (SAME).

A variável dependente foi a amputação e as variáveis independentes as condições clínicas de saúde dos idosos amputados (morbidades referidas em prontuário) e etiologia da amputação.

Montou-se um banco de dados e realizou-se análise quantitativa das informações, mediante processo sistematizado em base estatística. A análise dos dados foi processada utilizando-se o programa Epi Info for Windows, versão 3.5.1 e os resultados foram descritos por meio de medidas de frequência (absoluta e relativa), de tendência central e dispersão e foram apresentados por meio de tabelas segundo normas da ABNT: NBR 14724/11.

A pesquisa desenvolvida está vinculada ao projeto "FATORES ASSOCIADOS AS AMPUTAÇÕES DE MEMBROS SUPERIORES E INFERIORES EM IDOSOS”, o qual foi submetido e aprovado pelo Comitê de Ética em Pesquisa da Universidade Federal de Pernambuco (CAAE:10751619.1.0000.5208), atendendo às normas previstas na resolução 466/2012 do Conselho Nacional de Saúde - Ministério da Saúde.

\section{Resultados}

Em relação às comorbidades, verificou-se que a comorbidade mais prevalente é a Hipertensão Arterial Sistêmica (HAS) (83,7\%), seguida do Diabetes Mellitus (DM) $(83,3 \%)$, sendo a Osteoporose $(0,2 \%)$ e Osteoartose $(0,0 \%)$ menos observadas no público estudado. Ainda, foi realizado o teste de comparação de proporção, o qual foi significativo em todos os fatores avaliados ( $\mathrm{p}<0,05)$, exceto para outras comorbidades $(\mathrm{p}=0,845)$, indicando que o número de pacientes que possui outras comorbidades além das listadas é estatisticamente semelhante ao número de pacientes que não possui outras comorbidades. A Tabela 1 mostra a distribuição detalhada das comorbidades avaliadas nos pacientes.

Tabela 1. Distribuição das comorbidades avaliadas nos pacientes atendidos no Hospital Getúlio Vargas, Recife-PE, 2020.

\begin{tabular}{llll}
\hline Comorbidade avaliada & \multicolumn{2}{c}{ Resultado } & \multicolumn{2}{c}{ p-valor $^{\mathbf{1}}$} \\
\cline { 2 - 3 } & Ausente & Presente & $<0,001$ \\
\hline HAS* $^{*}$ & $68(16,3 \%)$ & $350(83,7 \%)$ & $<0,001$ \\
DM* $^{*}$ & $70(16,7 \%)$ & $348(83,3 \%)$ & $<0,001$ \\
Doenças cardiovasculares & $359(85,9 \%)$ & $59(14,1 \%)$ & $<0,001$ \\
Osteoporose & $417(99,8 \%)$ & $1(0,2 \%)$ & - \\
Osteoartose & $418(100,0 \%)$ & $0(0,0 \%)$ & $<0,001$ \\
Doenças neurológicas & $335(80,1 \%)$ & $83(19,9 \%)$ &
\end{tabular}




\begin{tabular}{|c|c|c|c|}
\hline Doenças respiratórias & $408(97,6 \%)$ & $10(2,4 \%)$ & $<0,001$ \\
\hline Neoplasia & $409(97,8 \%)$ & $9(2,2 \%)$ & $<0,001$ \\
\hline Outras & $211(50,5 \%)$ & $207(49,5 \%)$ & 0,845 \\
\hline
\end{tabular}

A Tabela 2 mostra a distribuição das outras comorbidades avaliadas nos pacientes que compuseram o estudo. Além disso, na Tabela 3, estão distribuídas as causas de amputação dos membros nos pacientes estudados.

\section{Discussão}

Os achados quanto ao número de idosos amputados com HAS foram semelhantes aos resultados encontrados após a busca na literatura. Um estudo com idosos amputados em um hospital de referência de Goiás constatou que do total de pacientes, 75\% tinham HAS 62\% eram portadores de DM (Brandão, et al., 2013). Semelhante a isso, o estudo de Adolfo et al. (2016), evidenciou que do total de pacientes amputados, $84 \%$ tinham hipertensão e $72 \%$ tinham diabetes.

Em contrapartida, apesar de muitos estudos constatarem essa prevalência, a maioria dá maior enfoque ao DM. Dados recentes mostram que cerca de 12,5 milhões de brasileiros são diabéticos, fazendo o país ocupar o quarto lugar entre os 10 países com o maior número de indivíduos com diabetes. Além disso, estima-se que a amputação é 10 a 20 vezes mais comuns na população com diabetes do que na população geral e que a cada 20 segundos uma pessoa tem uma amputação de membros por causa do diabetes (Sociedade Brasileira de Diabetes, 2015-2016).

Diversos fatores podem estar relacionados com o alto número de amputações em pessoas com DM, pois essa comorbidade, quando não controlada, pode causar diversas complicações, como Neuropatia Diabética (ND), cegueira, doenças cardiovasculares, Hipertensão Arterial Sistêmica (HAS), doença renal, entre outras. A amputação está intimamente ligada à ND, pois a polineuropatia distal sensitivo motora de membros inferiores representa a manifestação clínica mais comum desta complicação, resultando em perda de sensibilidade protetora e, como consequência, leva ao prejuízo da capacidade de reconhecimento de determinados estímulos como temperatura, pressão e dor ou mesmo a presença de ulcerações nos pés, caracterizando o "pé diabético" (Sociedade Brasileira de Diabetes, 2015-2016; Beaulieu, et al., 2015).

O pé diabético é considerado a principal causa de amputação do membro inferior (risco de 15 a 40 vezes maior). Mais do que uma complicação do DM, deve ser considerado um problema clínico complexo, que geralmente atinge o pé e/ou o tornozelo, e pode apresentar características clínicas variadas, tais como alterações de sensibilidade, presença de lesões, deformidades, alterações da marcha, infecções, entre outras, podendo muitas vezes ter como consequência a amputação. Desse modo, percebe-se a importância da adequada abordagem aos pés do idoso com DM e ações de educação em saúde para controle da doença, objetivando a prevenção de complicações e a restauração funcional da extremidade, tendo o indivíduo como protagonista do cuidado (Santos, et al., 2015).

Em contrapartida, muitos estudos evidenciam o pouco conhecimento de pessoas com diabetes acerca dos cuidados com os pés, bem como as falhas nas orientações oferecidas pela equipe de saúde (Borges, et al., 2015; Andrade, et al., 2010). Ademais, também existe a disposição do indivíduo para o autocuidado, fator importante para a prevenção de amputações (Policarpo, et al., 2014). Dessa forma, reforça-se a necessidade de equipes e serviços de saúde estabelecerem estratégias de educação em saúde sobre diabetes e suas particularidades, bem como a sensibilização do indivíduo para o autocuidado integrado. 
Ademais, um estudo de base populacional, constatou um risco de mortalidade dependente do tempo de diabetes após a amputação de membro inferior importante, que pode ser em parte resultado de um estilo de vida mais saudável em pacientes diabéticos ou do acesso a estruturas de tratamento específicas em indivíduos diabéticos (Andrés, et al., 2011).

É importante destacar que apesar de as doenças cardiovasculares não terem apresentado um percentual tão expressivo quanto outros achados, elas são a principal causa de óbito em escala global (Cubas, et al. 2013; Brasil, 2013). Portanto, uma estimativa de $14,1 \%$ em uma população de risco não deve ser ignorada na elaboração de políticas públicas.

Além disso, Nallegowda, et al (2012) constataram que o fatores de risco cardíaco significativos clínicos predominantes nos amputados são hipertensão $(89,5 \%)$, doença renal crônica $(31,6 \%)$, dislipidemia $(72,4 \%)$, e resistência à insulina associada ao diabetes.

Quanto à avaliação de outras comorbidades, observou-se que as mais frequentes são: Ex-tabagista (37,0\%), tabagista $(28,5 \%)$ e doença renal $(28,1 \%)$. As demais outras comorbidades citadas apresentaram prevalência menor que $2 \%$ e estão listadas na Tabela 2 .

Tabela 2. Distribuição de outras comorbidades nos pacientes atendidos no Hospital Getúlio Vargas, Recife-PE, 2020.

\begin{tabular}{lll}
\hline Outros tipos de comorbidades & $\mathbf{n}$ & $\%$ \\
\hline Ex-tabagista & 87 & 37,0 \\
Tabagista & 67 & 28,5 \\
Doença renal & 66 & 28,1 \\
Obesidade & 3 & 1,3 \\
Erisipela & 2 & 0,9 \\
Amaurose & 1 & 0,4 \\
\hline Ulcera em membro inferior crônica & 1 & 0,4 \\
Etilista & 1 & 0,4 \\
\hline Hipotireoidismo & 1 & 0,4 \\
\hline Neuropatia diabetica & 1 & 0,4 \\
\hline Anemiagite em membro inferior & 1 & 0,4 \\
\hline Retinopatia diabética & 1 & \\
\hline
\end{tabular}




\begin{tabular}{lcc} 
Dislipidemia & 1 & 0,4 \\
\hline TOTAL & 88 & 100,0 \\
\hline
\end{tabular}

*MMII: Membros Inferiores. Fonte: Autores (2021).

O tabagismo constitui fator de risco para o desenvolvimento de diversas condições clínicas, como doença cardiovascular, câncer, doença respiratória, doença renal, entre outras. Uma análise de regressão multivariada mostrou que o tabagismo foi um dos fatores de risco independentes para úlcera no pé, pois o tabaco representa um importante fator de risco cardiovascular, desencadeador de alterações endoteliais que comprometem a cicatrização e aumentam o risco de lesões. A história prévia dessa lesão corresponde a um fator de risco substancial para amputação não-traumática de membro inferior, devido ao seu potencial para infecção e seu grau de comprometimento do membro (Parisi, et al. 2016).

Além disso, sabe-se que o uso do tabaco causa estresse oxidativo celular, preditor de resistência insulínica e neuropatia diabética, também aumenta as taxas de DAP, diminuindo a capacidade de transporte de oxigênio, resultando em hipóxia tecidual, prejudicando, dessa forma, a cicatrização das lesões e aumentando o risco das amputações (Xia, et al., 2019). Portanto, o enfrentamento do tabagismo é crucial na prevenção do pé diabético e, por conseguinte, da amputação. Pesquisas recentes que avaliaram fatores de risco e condições associadas a amputação apresentaram um alto percentual de tabagismo, coincidindo com o encontrado neste estudo (Liu, et al., 2018; Formiga, et al., 2020).

Quanto à doença renal, diversos estudos mostram sua alta prevalência em idosos amputados. Uma pesquisa constatou que a insuficiência renal (Razão de risco (RR) 1,57, Intervalo de confiança (IC) 95\% 1,32-1,87), diabetes (RR 1,45, IC 95\% 1,32-1,60) e insuficiência cardíaca (RR 1,17, IC 95\% 1,05-1,31) associou-se independentemente a um aumento da taxa de amputação. Esse expressivo percentual de doença renal pode estar relacionado ao DM não controlado e à HAS, uma vez que a insuficiência renal pode ser desencadeada por ambas as condições clínicas (Pérez, et al., 2019).

Além disso, o DM apresenta complicações micro e macrovasculares, sendo a principal causa de insuficiência renal em estágio avançado, cegueira, amputação de membro inferior e doença cardiovascular (DCV) (Lazzarini, et al., 2012). Essas complicações afetam diretamente a qualidade de vida relacionada à saúde de um indivíduo, a utilização de serviços de saúde, os custos e a expectativa de vida.

A descrição da doença renal albergou os pacientes com Doença Renal Crônica (DRC), condição que muitas vezes requer tratamento dialítico. O estudo de coorte retrospectivo de pacientes dependentes de diálise e sem diálise submetidos a amputação de membros concluiu que a readmissão ou morte após amputação é aumentada entre pacientes em diálise. As taxas de mortalidade pós-operatória em 30 dias (15\% vs 7\%) e as taxas de readmissão (35\% vs $20 \%$ por 30 pessoas-dia; ambas P <0,001) foram maiores nos pacientes em diálise (Hickson, et al. 2018).

Estudos internacionais recentes identificaram a diálise como um fator de risco independente para úlceras nos pés. Esses estudos também identificaram que as amputações de membro são um evento frequente em pacientes em terapia hemodialítica (TH) e que esse risco aumenta ainda mais com a presença de DM simultâneo (Ndip, et al., 2010; Meyer, et al., 2016).

Vale a pena destacar que a literatura aponta para fatores associados a readimissões e reamputações de pacientes, tais como internação eletiva, doença arterial periférica (DAP), doença renal crônica, infecção e isquemia, e observou que a comorbidade do paciente influencia diretamente o risco de readmissão do paciente, bem como o risco de reamputação e mortalidade (Combe, et al., 2009).

Em relação às causas de amputação nos pacientes estudados, verificou-se que a causa mais prevalente foi Isquemia Crítica de Membro Inferior Direito (ICMID) (31,5\%), seguido de Isquemia Crítica de Membro Inferior Esquerdo (ICMIE) 
$(28,3 \%)$ e gangrena úmida $(14,4 \%)$. As causas menos citadas são: amputação traumática, ferimento por arma de fogo, fratura exposta, SEPSE e IMSE, as quais têm suas prevalências mostradas na Tabela 3.

Tabela 3. Distribuição das causas de amputação nos pacientes atendidos no Hospital Getúlio Vargas, Recife-PE, 2020.

\begin{tabular}{|c|c|c|}
\hline Causas & $\mathbf{n}$ & $\%$ \\
\hline ICMID* & 177 & 31,5 \\
\hline ICMIE* & 159 & 28,3 \\
\hline Gangrena úmida & 81 & 14,4 \\
\hline Infecção/necrose/abcesso & 59 & 10,5 \\
\hline Gangrena gasosa & 37 & 6,6 \\
\hline Pe diabético & 22 & 3,9 \\
\hline Gangrena não especificada & 10 & 1,8 \\
\hline $\mathrm{OAA}^{*}$ & 7 & 1,2 \\
\hline Osteomielite & 3 & 0,5 \\
\hline Linfagite & 2 & 0,4 \\
\hline Amputação traumática & 1 & 0,2 \\
\hline Ferimento por arma de fogo & 1 & 0,2 \\
\hline Fratura exposta & 1 & 0,2 \\
\hline SEPSE & 1 & 0,2 \\
\hline IMSE* & 1 & 0,2 \\
\hline TOTAL & 562 & 100,0 \\
\hline
\end{tabular}

*ICMID: Isquemia Crítica de Membro Inferior Direito; ICMIE: Isquemia Crítica de Membro Inferior Esquerdo; OAA: Oclusão Arterial Aguda; IMSE: Isquemia de Membro Superior Esquerdo. Fonte: Autores (2021).

Dentre as causas de amputação, a isquemia crítica de membro inferior (ICMI) foi a principal, correspondendo a $31,5 \%$ no membro direito e 28,3\% no membro esquerdo. Essa condição representa a forma mais avançada de doença arterial periférica (DAP) e é definida como dor crônica em repouso isquêmica, úlceras ou gangrena atribuível à doença oclusiva arterial. Diversos estudos relacionam a ICMI a alto risco de amputação, seja ela menor ou maior (Ward, et al., 2017; Foley, et al., 2016; Howard, et al., 2015). Além disso, uma pesquisa recente de base populacional no Reino Unido descobriu que as principais taxas de sobrevida livre de amputação 5 anos após o diagnóstico de ICMI eram de apenas 27\% (Foley \& Armstrong \& Waldo, 2016). 
Ademais, pacientes submetidos a amputação menor por ICMI também apresentam alto risco de amputação maior subsequente: aos 5 anos, 8,4\% apresentaram amputação maior do membro contralateral e 14,2\% sofreram amputação maior no mesmo membro. Um estudo anterior em um único centro estimou a taxa de re-amputação em 1 ano em 27\% (Howard, et al., 2015). O que pode estar relacionado ao alto número de amputações por ICMI é que dentre os fatores de risco para a DAP e, consequentemente, para a ICMI, estão o DM e o tabagismo (Armstrong, et al., 2017). Como foi evidenciado, a maioria dos idosos amputados era tabagista ou ex-tabagista e era portador de DM.

A pesquisa de Kelly, et al. (2017) mostrou que um total de 2.552.541 pacientes tiveram pelo menos um diagnóstico de DAP. Destes, 226.747 (9\%) possuíam um registro da ICMI. Além disso, essa condição continua sendo a indicação mais comum para a amputação de membros inferiores, com tabagismo, hipertensão e diabetes sendo as principais doenças comórbidas associadas.

A gangrena úmida, por sua vez, também apresentou um percentual significativo como causa de amputação (14,4\%). Essa condição é caracterizada pela necrose de uma parte do corpo devido à falta de circulação, lesão ou infecção. Geralmente, decorre de invasão da região necrosada por microrganismos anaeróbios produtores de enzimas que tendem a liquefazer os tecidos acometidos (Mahesh \& Mallappa \& Vithoulkas, 2015).

As gangrenas estão muito associadas a complicações do diabetes, fazendo parte do que compõe o "pé diabético". As alterações isquêmicas ocorridas no diabético, decorrem de inúmeras disfunções vasculares frente ao quadro de hiperglicemia. Isso predispõe, principalmente, ao desenvolvimento da gangrena do tipo seca, que está usualmente associada à necrose isquêmica de extremidades; sendo que para isso, fatores como: drenagem insuficiente e perda de líquido tecidual por evaporação contribuem para o padrão morfológico de apresentação (Datasus, 2019; Borges, 2017).

Porém outros tipos de gangrena também podem estar associados, como a gangrena úmida. Desse modo, caso o pé diabético apresente algum tipo de ulceração ou descontinuidade da pele, e seja infectado por micro-organismos, existe a possibilidade do desenvolvimento destes dois padrões de gangrena (Borges, 2017).

O estudo de Souza, et al. (2019) constatou que que a gangrena foi considerada como a causa da maioria das amputações tanto nas relacionadas a afecções clínicas como nas amputações traumáticas, sendo as gangrenas úmida e gasosa as mais prevalentes.

Torna-se necessário destacar que a gangrena é frequentemente associada ao DM, sendo 100 vezes mais comum nos indivíduos diabéticos do que na população em geral, uma vez que a DM induz a hipercolesterolemia, a qual aumenta o risco de aterosclerose e consequentemente a gangrena nas extremidades (Kumar \& Abbas \& Aster, 2010).

\section{Conclusão}

Portanto, considerando o objetivo do estudo e os resultados obtidos, evidenciou-se que as comorbidades mais prevalentes em idosos amputados foram a Hipertensão Arterial Sistêmica (HAS), o Diabetes Mellitus (DM), ex-tabagismo, tabagismo e doença renal. Além disso, quanto às etiologias das amputações, destacou-se a Isquemia Crítica de Membro Inferior (ICMI) direita e esquerda, bem como a gangrena úmida. Então, espera-se que o estudo possa inspirar outras pesquisas estatísticas para que se conheça realmente o perfil da população envolvida e suas necessidade, além de corroborar para a elaboração de estratégias educativas e preventivas, bem como contribuir para que ações de educação em saúde possam ser direcionadas para esse público a fim de gerar hábitos saudáveis e manter uma assistência adequada com foco no controle e tratamento dos fatores causais das amputações, objetivando a diminuição desse índice e melhoria da qualidade de vida. 


\section{Referências}

Andrade, N. H. S. de, Sasso-Mendes, K. D., Faria, H. T. G., Martins, T. A., Santos, M. A. dos, Teixeira, C. R. de S., \& Zanetti, M. L. (2010). Pacientes com diabetes mellitus: cuidados e prevenção do pé diabético em atenção primária à saúde. Rev. Enferm. UERJ, 18(4), 616-621.

Armstrong, E. J., Ryan, M. P., Baker, E. R., Martinsen, B. J., Kotlarz, H., \& Gunnarsson, C. (2017). Risk of major amputation or death among patients with critical limb ischemia initially treated with endovascular intervention, surgical bypass, minor amputation, or conservative management. Journal of medical economics, 20(11), 1148-1154. doi: 10.1080/13696998.2017.1361961

Baubeta Fridh, E., Andersson, M., Thuresson, M., Sigvant, B., Kragsterman, B., Johansson, S., Hasvold, P., Nordanstig, J., \& Falkenberg, M. (2018). Editor's Choice - Impact of Comorbidity, Medication, and Gender on Amputation Rate Following Revascularisation for Chronic Limb Threatening Ischaemia. European journal of vascular and endovascular surgery : the official journal of the European Society for Vascular Surgery, 56(5), 681-688. Recuperado em 26 de Setembro de 2021 por doi: 10.1016/j.ejvs.2018.06.003

Beaulieu, R. J., Grimm, J. C., Lyu, H., Abularrage, C. J., \& Perler, B. A. (2015). Rates and predictors of readmission after minor lower extremity amputations. Journal of vascular surgery, 62(1), 101-105. doi: 10.1016/j.jvs.2015.02.021

Borges, A.M.F., Vargas, M.A.O., Schoeller, S.D., Kinoshita, E.Y., Ramos, F.R.S., \& Lima, D.K.S. (2015). Cirurgias de amputação realizadas em hospitais públicos de referência. Rev Enferm UFPE online , 9(Supl. 7), 9053-61. doi: 10.5205/reuol.8074-70954-1-SM0907supl201515

Borges, W. R. (2017). Fatores De Risco Para Amputação Em Pacientes Com Isquemia Crítica Crônica Dos Membros Inferiores. J Vasc Bras. 16(2), 180-181. doi: $10.1590 / 1677-5449.003317$

Brandão, M.L., Júnior, G.B.P., Barbosa, L.A, \& Rocha, V.A.F. (2013). Amputações maiores dos membros inferiores por causa vascular / Major lower limb amputation for vascular condition. Sociedade Brasileira de Angiologia e Cirurgia Vascular - RJ. http://sbacvrj.com.br/novo/artigo/amputacoes-maiores-dosmembros-inferiores-por-causa-vascular-major-lower-limb-amputation-for-vascular-condition/

Brasil. Ministério da Saúde. (2013). Diretrizes de atenção a pessoa amputada. Secretaria de Atenção à Saúde. Departamento de Ações Programáticas Estratégicas. 1. ed. 1. reimp. - Brasília, DF: Ministério da Saúde.

Centro Colaborador da OMS para a Classificação de Doenças em Português - CBCD. (2008). Classificação Estatística Internacional de Doenças e Problemas Relacionados à Saúde - CID-10. (10. ed., Vol. 1). In: Departamento de Informática do SUS - DATASUS. São Paulo: Faculdade de Saúde Pública, Universidade de São Paulo. [citado 15 jan 2019]. http://www.datasus.gov.br/cid10/V2008/cid10.html

Chamlian, T. R., Varanda, R. dos R., Pereira, C. L., Resende, J. M. de, \& Faria, C. C. de. (2013). Epidemiological profile of lower limb amputees patients assisted at the Lar Escola São Francisco between 2006 and 2012. Acta Fisiátrica, 20(4), 219-223. doi:10.5935/0104-7795.20130036

Combe, C., Albert, J. M., Bragg-Gresham, J. L., Andreucci, V. E., Disney, A., Fukuhara, S., Goodkin, D. A., Gillespie, B. W., Saito, A., Jadoul, M., \& Pisoni, R. L. (2009). The burden of amputation among hemodialysis patients in the Dialysis Outcomes and Practice Patterns Study (DOPPS). American journal of kidney diseases : the official journal of the National Kidney Foundation, 54(4), 680-692. doi: 10.1053/j.ajkd.2009.04.035

Cubas, M. R., Santos, O. M. dos, Retzlaff, E. M. A., Telma, H. L. C., Andrade, I. P. S. de, Moser, A. D. de L., \& Erzinger, A. R. (2013). Pé diabético: orientações e conhecimento sobre cuidados preventivos. Fisioterapia Em Movimento, 26(3), 647-655. doi: 10.1590/s0103-51502013000300019

Andrés, A.L., Huedo, M. A. M, Garrido, P. C., Barrera, V. H., Miguel, A. G., \& García, R. J. (2011). Trends in Lower-Extremity Amputations in People With and Without Diabetes in Spain. American Diabetes Association: Diabetes Care, 34(7), 1570-1576. doi: 10.2337/dc11-0077

Almeida, R. M., Perez, R. R., \& Da Rosa, L. M. A. (2013). Prevalência de Amputações no Hospital Escola da Cidade de Itajubá no Período entre 1999 e 2012/ Prevalence of Amputations in the School Hospital of Itajubá between 1999 and 2012. Revista Ciências em Saúde, 3(2), 34-42. doi: 10.21876/rcs fmit.v3i2.253

de Jesus-Silva, S. G., de Oliveira, J. P., Brianezi, M., Silva, M., Krupa, A. E., \& Cardoso, R. S. (2017). Análise dos fatores de risco relacionados às amputações maiores e menores de membros inferiores em hospital terciário. Jornal vascular brasileiro, 16(1), 16-22. doi: 10.1590/1677-5449.008916

Departamento de Informática do SUS - DATASUS. (2019). Informações de Saúde (TABNET). Brasília, DF: Ministério da Saúde; [citado 15 fev 2018]. http://tabnet.datasus.gov.br/cgi/tabcgi.exe?sih/cnv/qiuf.def

Dillon, M. P., Fortington, L. V., Akram, M., Erbas, B., \& Kohler, F. (2017). Geographic Variation of the Incidence Rate of Lower Limb Amputation in Australia from 2007-12. PloS one, 12(1), e0170705. doi: 10.1371/journal.pone.0170705

Diretrizes da Sociedade Brasileira de Diabetes (2015-2016) / Adolpho Milech. [et. al.]; organização José Egidio Paulo de Oliveira, Sérgio Vencio - São Paulo: A.C. Farmacêutica.

Doenças cardiovasculares - OPAS/OMS | Organização Pan-Americana da Saúde. (n.d.). Www.paho.org. Retrieved September 20, 2021, from https://www.paho.org/bra/index.php?option=com_content\&view=article\&id=5253:doencas-cardiovasculares\&Itemid=1096

Estimativa Sociedade Brasileira de Angiologia e de Cirurgia Vascular - SBACV. (2018). https://sbacv.org.br/imprensa/estimativas/

Foley, T. R., Armstrong, E. J., \& Waldo, S. W. (2016). Contemporary evaluation and management of lower extremity peripheral artery disease. Heart (British Cardiac Society), 102(18), 1436-41. doi:10.1136/heartjnl-2015-309076

Hickson, L. J., Rule, A. D., Thorsteinsdottir, B., Shields, R. C., Porter, I. E., Fleming, M. D., Ubl, D. S., Crowson, C. S., Hanson, K. T., Elhassan, B. T., Mehrotra, R., Arya, S., Albright, R. C., Williams, A. W., \& Habermann, E. B. (2018). Predictors of early mortality and readmissions among dialysis patients undergoing lower extremity amputation. Journal of vascular surgery, 68(5), 1505-16. doi: 10.1016/j.jvs.2018.03.408 
Howard, D. P., Banerjee, A., Fairhead, J. F., Hands, L., Silver, L. E., Rothwell, P. M., \& Oxford Vascular Study (2015). Population-Based Study of Incidence, Risk Factors, Outcome, and Prognosis of Ischemic Peripheral Arterial Events: Implications for Prevention. Circulation, 132(19), 1805-15. doi: 10.1161/CIRCULATIONAHA.115.016424

Icks, A., Scheer, M., Morbach, S., Genz, J., Haastert, B., Giani, G., Glaeske, G., \& Hoffmann, F. (2011). Time-dependent impact of diabetes on mortality in patients after major lower extremity amputation: survival in a population-based 5-year cohort in Germany. Diabetes care, 34(6), 1350-54. doi: doi.org/10.2337/dc10-2341

Kelly, D. A., Pedersen, S., Tosenovsky, P., \& Sieunarine, K. (2017). Major Lower Limb Amputation: Outcomes are Improving. Annals of vascular surgery, 45, 29-34. doi:10.1016/j.avsg.2017.05.039

Kumar V, Abbas AK, Aster JC. Robbins \& Cotran Patologia. (2010). Bases Patológicas das Doenças. (8. ed.) Rio de Janeiro: Elsevier

Lazzarini, P. A., Gurr, J. M., Rogers, J. R., Schox, A., \& Bergin, S. M. (2012). Diabetes foot disease: the Cinderella of Australian diabetes management?. Journal of foot and ankle research, 5(1), 24. doi:10.1186/1757-1146-5-24

Leandro, G., Parolim, S. C., Moro, C., \& Carvalho, D. R. (2018). Mineração de dados na avaliação de óbitos após cirurgia de amputação. Jornal vascular brasileiro, 17(1), 10-18. doi:10.1590/1677-5449.008317

Lima-Costa, M. F., Barreto, S. M. (2003). Tipos de estudos epidemiológicos: conceitos básicos e aplicações na área do envelhecimento. Epidemiologia e Serviços de Saúde, 12(4), 189-201. http://scielo.iec.gov.br/pdf/ess/v12n4/v12n4a03.pdf

Liu, M., Zhang, W., Yan, Z., \& Yuan, X. (2018). Smoking increases the risk of diabetic foot amputation: A meta-analysis. Experimental and therapeutic medicine, 15(2), 1680-85. doi:10.3892/etm.2017.5538

Mahesh S, Mallappa M \& Vithoulkas G. (2015). Gangrene: Five case studies of gangrene, preventing amputation through Homoeopathic therapy. Indian J Res Homoeopathy 9(2), 114-22. doi:10.4103/0974-7168.159544

Meyer, A., Lang, W., Borowski, M., Torsello, G., Bisdas, T., \& CRITISCH collaborators (2016). In-hospital outcomes in patients with critical limb ischemia and end-stage renal disease after revascularization. Journal of vascular surgery, 63(4), 966-73. doi: 10.1016/j.jvs.2015.10.009

Montiel, A., Vargas, M. A. de O., \& Leal, S. M. C. (2012). Caracterização de pessoas submetidas à amputação. Enfermagem Em Foco, 3(4), 169-73. doi: 10.21675/2357-707X.2012.v3.n4.377

Nallegowda, M., Lee, E., Brandstater, M., Kartono, A. B., Kumar, G., \& Foster, G. P. (2012). Amputation and cardiac comorbidity: analysis of severity of cardiac risk. $P M$ \& $R$ : the journal of injury, function, and rehabilitation, 4(9), 657-66. https://doi.org/10.1016/j.pmrj.2012.04.017

Ndip, A., Rutter, M. K., Vileikyte, L., Vardhan, A., Asari, A., Jameel, M., Tahir, H. A., Lavery, L. A., \& Boulton, A. J. (2010). Dialysis treatment is an independent risk factor for foot ulceration in patients with diabetes and stage 4 or 5 chronic kidney disease. Diabetes care, 33(8), 1811-1816. doi: 10.2337/dc100255

Oliveira, C. D. L., Rocha, K. S. C., Costa, E. M., Almeida, R. C., Faria, M. L., Aquino, J. A., Cabral, H. K., Barbosa-Júnior, A. R., Sakamoto, R. Y., \& Cardoso, C. S. (2017). Perfil clínico do paciente diabético após internação devido a complicação por condição sensível à atenção primária. Revista Da Faculdade de Ciências Médicas de Sorocaba, 19(3), 139. doi: 10.23925/1984-4840.2017v19i3a8

Parisi, M. C., Moura Neto, A., Menezes, F. H., Gomes, M. B., Teixeira, R. M., de Oliveira, J. E., Pereira, J. R., Fonseca, R. M., Guedes, L. B., Costa E Forti, A., de Oliveira, A. M., de Medeiros Nóbrega, M. B., Colares, V. N., Schmid, H., Nienov, O. H., Nery, M., Fernandes, T. D., Pedrosa, H. C., Schreiber de Oliveira, C., Ronsoni, \& Saad, M. J. (2016). Baseline characteristics and risk factors for ulcer, amputation and severe neuropathy in diabetic foot at risk: the BRAZUPA study. Diabetology \& metabolic syndrome, 8, 25. doi: 10.1186/s13098-016-0126-8

Peek M. E. (2011). Gender differences in diabetes-related lower extremity amputations. Clinical orthopaedics and related research, 469(7), 1951-55. doi: $10.1007 / \mathrm{s} 11999-010-1735-4$

Pérez, D. S. B., Pilar, M. S. M., Liseth, R. S., Giovanni, J. B. W. (2019) Determinantes sociales de la salud influyentes en la complicación de pacientes hipertensos y diabéticos que padecen insuficiencia renal. Rev. Méd. Risaralda, 25(1) 15-21. doi: 10.22517/25395203.15871

Pinheiro Fabricio Formiga, N., Alves Firmino, P. R., Félix Rebouças, V. de C., Oliveira, C. J., Moura de Araújo, M. F., \& Parente Garcia Alencar, A. M. (2020). estratificação de risco para pé diabético numa população de idosos acompanhados na atenção primária. Revista Baiana de Enfermagem34, . doi: $10.18471 /$ rbe.v34.34097

Policarpo, N. de S., Moura, J. R. A., Melo Júnior, E. B. de, Almeida, P. C. de, Macêdo, S. F. de, \& Silva, A. R. V. da. (2014). Knowledge, attitudes and practices for the prevention of diabetic foot. Revista Gaúcha de Enfermagem, 35(3), 36-42. doi: 10.1590/1983-1447.2014.03.45187

Rolim, D., Sampaio, S., Gonçalves-Dias, P., Almeida, P., Almeida-Lopes, J., \& Teixeira, J. F. (2015). Mortalidade depois da amputação. Angiologia E Cirurgia Vascular, 11(3), 166-70. doi: 10.1016/j.ancv.2015.06.001

Santos, B. K. dos, Luz, S. C. T. da, Santos, K. B. dos, Honório, G. J. da S., \& Farias, G. de O. (2018). Atuação de equipe multiprofissional no atendimento à pessoa amputada: contextualizando serviços e protocolos hospitalares. Cadernos Brasileiros de Terapia Ocupacional, 26(3), 527-37. doi: 10.4322/25268910.ctoao1193

Santos, I. C. R. V., Carvalho, E. F. de, Souza, W. V. de, \& Albuquerque, E. C. de. (2015). Factors associated with diabetic foot amputations. Jornal Vascular Brasileiro, 14(1), 37-45. doi: 10.1590/1677-5449.20140049 
Research, Society and Development, v. 10, n. 12, e533101220890, 2021

(CC BY 4.0) | ISSN 2525-3409 | DOI: http://dx.doi.org/10.33448/rsd-v10i12.20890

Santos, K., Luz, S., Mochizuki, L., \& d'Orsi, E. (2018). Burden of disease from lower limb amputations attributable to diabetes mellitus in Santa Catarina State, Brazil, 2008-2013. Carga da doença para as amputações de membros inferiores atribuíveis ao diabetes mellitus no Estado de Santa Catarina, Brasil, 2008-2013. Cadernos de saúde pública, 34(1). doi: 10.1590/0102-311X00013116

Schmidt, M. I., Duncan, B. B., e Silva, G. A., Menezes, A. M., Monteiro, C. A., Barreto, S. M., Chor, D., \& Menezes, P. R. (2011). Chronic non-communicable diseases in Brazil: burden and current challenges. The Lancet, 377(9781), 1949-61. doi: 10.1016/s0140-6736(11)60135-9

Seidel, A. C., Nagata, A. K., Almeida, H. C. de, \& Bonomo, M. (2009). Epistemologia sobre amputações e desbridamentos de membros inferiores realizados no Hospital Universitário de Maringá. Jornal Vascular Brasileiro, 7(4), 308-15. doi: 10.1590/s1677-54492009005000002

Senefonte, F. R. de A., Rosa, G. R. de P. S., Comparin, M. L., Covre, M. R., Jafar, M. de B., Andrade, F. A. M. de, Maldonado Filho, G., \& Nogueira Neto, E. (2012). Amputação primária no trauma: perfil de um hospital da região centro-oeste do Brasil. Jornal Vascular Brasileiro, 11(4), 269-76. doi: 10.1590/s167754492012000400004

Souza, Y. P. de, Santos, A. C. O. dos, \& Albuquerque, L. C. de. (2019). Caracterização das pessoas amputadas de um hospital de grande porte em Recife (PE, Brasil). Jornal Vascular Brasileiro, 18. doi: 10.1590/1677-5449.190064

Ward, R., Dunn, J., Clavijo, L., Shavelle, D., Rowe, V., \& Woo, K. (2017). Outcomes of Critical Limb Ischemia in an Urban, Safety Net Hospital Population with High WIfI Amputation Scores. Annals of vascular surgery, 38, 84-89. doi: 10.1016/j.avsg.2016.08.005

WHO. World Health Organization. (2005). Envelhecimento ativo: uma política de saúde. Tradução Suzana Gontijo. Brasília: Organização Pan-Americana da Saúde, 60p. https://bvsms.saude.gov.br/bvs/publicacoes/envelhecimento_ativo.pdf

Xia, N., Morteza, A., Yang, F., Cao, H., \& Wang, A. (2019). Review of the role of cigarette smoking in diabetic foot. Journal of diabetes investigation, 10(2), 202-15. doi:10.1111/jdi.12952 